\title{
Artificial Neural Network Analysis of Xinhui Pericarpium Citri Reticulatae Using Gas Chromatography - Mass Spectrometer - Automated Mass Spectral Deconvolution and Identification System
}

\author{
Xiaoqun Ou ${ }^{1}$, Hao $\mathrm{Li}^{2}$, Xiumei Yang ${ }^{1}$, Maolan $\mathrm{Tan}^{1}$, Hui Ao ${ }^{1}$ and Jin Wang ${ }^{1 *}$ \\ ${ }^{1}$ College of Pharmacy, Chengdu University of Traditional Chinese Medicine, ${ }^{2}$ College of Chemistry, Sichuan University, \\ Chengdu, Sichuan 610064, China
}

*For correspondence: Email: wangjin0816@126.com; Tel: + 86-13880900787

Received: 6 May 2015

Revised accepted: 5 October 2015

\begin{abstract}
Purpose: To develop an effective analytical method to distinguish old peels of Xinhui Pericarpium citri reticulatae (XPCR) stored for $>3$ years from new peels stored for $<3$ years.

Methods: Artificial neural networks (ANN) models, including general regression neural network (GRNN) and multi-layer feedforward neural network (MLFN), were used to analyze the Gas Chromatography Mass Spectrometer - Automated Mass Spectral Deconvolution and Identification System (GC-MSAMDIS) data of the essential oils of the XPCR. The Root Mean Square (RMS) errors of each ANN model was obtained through judging the characteristic of old peels and new peels.

Results: The Root Mean Square (RMS) error of GRNN was 0.22, less than the error MLFN at different levels, indicating that GRNN model is more reliable and accurate for judging the characteristics of old peels and new ones.

Conclusion: The general regression neural network model is established to reliably distinguish between old peels and new peels.
\end{abstract}

Keywords: Artificial neural networks, Xinhui, Pericarpium, Citri reticulatae, Gas Chromatography, Automated Mass Spectral Deconvolution and Identification System, Peels

Tropical Journal of Pharmaceutical Research is indexed by Science Citation Index (SciSearch), Scopus, International Pharmaceutical Abstract, Chemical Abstracts, Embase, Index Copernicus, EBSCO, African Index Medicus, JournalSeek, Journal Citation Reports/Science Edition, Directory of Open Access Journals (DOAJ), African Journal Online, Bioline International, Open-J-Gate and Pharmacy Abstracts

\section{INTRODUCTION}

Pericarpium citri reticulatae (PCR) is the dried peel of Citrus reticulata Blanco, commonly known as tangerine or orange, and its cultivars. Xinhui Pericarpium citri reticulatae (XPCR), which derived from Citrus reticulata 'Chachi', was regarded as the best national product of geoherbalism since ancient times. According to the number of years of storage, PCR can be divided into two types: 'new peels' and 'old peels'. Peels that have been stored for more than
3 years are known as old peels, while those stored for less than three years are known as new peels. According to clinical experience, the longer XPCR was stored, the better its effect is. However, it is difficult to identify the number of years of storage from the appearance of the peels (Fig 1), in addition most of the old peels are adulterated new peels during processing.

In the past few decades, much work has been done using PCR to identify the storage year [1,2]. However, all these works show certain 
drawbacks without applying further exploratory data analysis to identify accurately the number of years the peels have been stored. Essential oil is one of the major effective components of XPCR and it is complicated. The ingredients in the oil exist in a nonlinear relationship, which is very suitable for artificial neural networks.

Pattern recognition through neural networks is an automatic processing and interpretation approach which uses mathematical techniques on computer [3]. It is envisioned that artificial neural networks (ANN) can be an alternative approach to distinguish between old peels and new peels. In this article, two artificial neural networks (ANNs) models-the general regression neural network (GRNN) and multilayer feedforward neural network (MLFN) were used to analyze and interpret the GC-MS-AMDIS data of the essential oils of XPCR with different storage time. Thirty five batches of authentic XPCRs - 21 batches with 12 months of storage time and 14 batches stored for 11 years- were collected to study the complicated and effective constituents with the use of GC-MS. The collected GC-MS data were then analyzed by two ANNs models to confirm the authenticity of the storage time of each batch. The model with smaller RMS errors was confirmed as the reliable judge for distinguishing 'old peels' from 'new peels'.
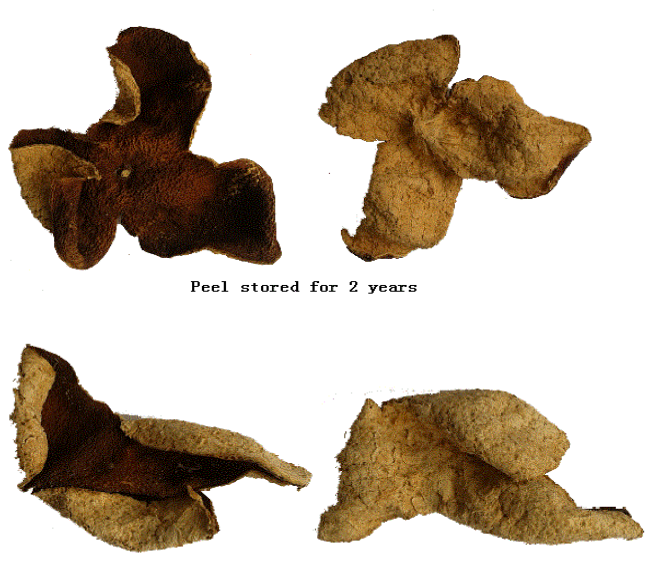

Peel stored for 5 years

\section{EXPERIMENTAL}

\section{Materials and sample preparation}

$\mathrm{N}$-hexane (HPLC grade) was purchased from Fisher Scientific. Anhydrous sodium sulfate (analytical reagent) was acquired from Chengdu Kelon Co. Ltd. Thirty five batches of XPCR samples were collected from the Xinhui District, Guangdong Province. Through an identification process developed by Dr. Xianming Lu (Professor of Medicine Specimen Center of Chengdu University of Traditional Chinese Medicine), 21 batches of the peels were new and had been stored for at least 12 months; the remaining 14 batches were else old and had been stored for more than 4 years.

\section{Extraction of essential oils}

The samples were cut into small pieces $(5 \mathrm{~mm} \times$ $5 \mathrm{~mm}$ ). In a typical experiment, $20 \mathrm{~g}$ samples were put in a round-bottom flask and steam distilled for 6 hours. The essential oils were collected and diluted by $n$-hexane, and then transferred to auto-sampler vials.

\section{Analysis of the essential oils by GC-MS- AMDIS}

The oils were analyzed by an Agilent 7890A5975C instrument equipped with a quartz capillary column $(0.25 \mathrm{~mm} \times 30 \mathrm{~m}, 0.25 \mu \mathrm{m})$ and an Agilent $5975 \mathrm{C}$ MS detector. Temperature programmes are listed in Table 1 . One $\mu \mathrm{L}$ of each sample was injected into GC-MS with a split ratio of 10:1. The temperature of the injection port and the detector were both $270{ }^{\circ} \mathrm{C}$. High purity helium (> $99 \%$ ) was used as the carrier gas with a flow rate of $1 \mathrm{~mL} / \mathrm{min}$. All data were obtained by collecting the full-scan mass spectra within the scan range 30-550 amu. The database was NIST08s.L (National Institute of Standards and Technology). According to the mass spectrometer coupled with the automated mass spectral deconvolution and identification system (AMDIS) as well as previous studies, the presence or absence of individual components were all recorded for an ANN analysis.

Fig 1: Peels stored for 2 years versus 5 years

Table 1: Temperature program

\begin{tabular}{ccccc}
\hline Ramp & $\begin{array}{c}\text { Initial } \\
\text { temperature } \\
\left({ }^{\circ} \mathbf{C}\right)\end{array}$ & $\begin{array}{c}\text { Rate of temperature } \\
\text { increase } \\
\left({ }^{\circ} \mathbf{C} / \mathbf{m i n}\right)\end{array}$ & $\begin{array}{c}\text { Final temperature } \\
\left({ }^{\circ} \mathbf{C}\right)\end{array}$ & $\begin{array}{c}\text { Hold time } \\
(\mathbf{m i n})\end{array}$ \\
\hline $\begin{array}{c}\text { First } \\
\text { Second }\end{array}$ & - & - & - & - \\
Third & - & 1 & 80 & 10 \\
Forth & - & 10 & 150 & 0 \\
\hline
\end{tabular}




\section{Statistical analysis}

To quantify the results of the model, the judgments generated by ANN model were presented as "1" or "0". "1" represents the characteristics of new peels while " 0 " represents the characteristics of old peels. For computerbased experiments, NeuralTools $\AA$ Software (Trial Version, Palisade Corporation, NY, USA) was used to develop ANN models. The chemical components were set as independent variables while the quantified results $(0$ or 1$)$ were taken as dependent variables. According to saliency analysis by Microsoft Excel, the level of statistical significance between new peels and old peels is less than 0.04 , indicating that there is significant difference between the two kinds of samples in this study.

\section{RESULTS}

The GC-MS-AMDIS results of the 35 samples showed that 31 compounds were present, and the relative contents of the components were more than $90 \%$ of the total content of 34 compounds. The main compounds were myrcene, 4-carene, d-limonene, g-terpinene, terpinolene, linalool, a-terpineol, methyl 2(methylamino) benzoate, a-sinensal, $\mathrm{n}$ hexadecanoic acid and d-limonene. Therefore, the contents of all the compounds were used as independent variables in the training of ANN models.

\section{Model development}

Two types of ANN models were used to establish the developed models for the peels: the general regression neural network (GRNN) model and the multi-layer feedforward neural network (MLFN) model. With the MLFN model, in order to find out the best number of nodes, different nodes were set from 2 to 20 . The target of the first selection was to find out the best model of the judgment. Therefore, 28 sample groups were used as the trained set, while 7 sample groups were the tested set. The results are shown in Table 2.

According to the RMS errors of the different models in Table 2, it is obvious that the GRNN model is much better than the MLFN models in distinguishing old peels from new peels. In order to make a comparison between the different nodes of the MLFN models, a scatter diagram was obtained in Fig 2.
From Fig 2, it can be observed that the RMS error of the MLFN model is unstable and can be concluded by linear regression. The fluctuations of the RMS errors show that the MLFN models are less robust than the GRNN model during the classification process. In addition, the running time of different models shown in Table 2 indicate that the time for training MLFN models is much longer than those for GRNN model.

Table 2: The results of different ANN models for distinguishing new from old peels ${ }^{a}$

\begin{tabular}{lcc}
\hline ANN model & RMS error & Training time \\
\hline GRNN & 0.22 & $0: 00: 00$ \\
MLFN 2 Nodes & 0.65 & $0: 02: 40$ \\
MLFN 3 Nodes & 0.53 & $0: 02: 06$ \\
MLFN 4 Nodes & 0.53 & $0: 02: 17$ \\
MLFN 5 Nodes & 0.53 & $0: 02: 12$ \\
MLFN 6 Nodes & 0.65 & $0: 02: 55$ \\
MLFN 7 Nodes & 0.72 & $0: 02: 32$ \\
MLFN 8 Nodes & 0.65 & $0: 03: 03$ \\
MLFN 9 Nodes & 0.65 & $0: 04: 18$ \\
MLFN 10 Nodes & 0.53 & $0: 03: 55$ \\
MLFN 11 Nodes & 0.65 & $0: 05: 01$ \\
MLFN 12 Nodes & 1.18 & $0: 05: 58$ \\
MLFN 13 Nodes & 1.01 & $0: 04: 31$ \\
MLFN 14 Nodes & 0.92 & $0: 06: 18$ \\
MLFN 15 Nodes & 0.55 & $0: 07: 31$ \\
MLFN 16 Nodes & 0.63 & $0: 05: 06$ \\
MLFN 17 Nodes & 0.36 & $0: 47: 15$ \\
MLFN 18 Nodes & 1.31 & $0: 09: 55$ \\
MLFN 19 Nodes & 1.49 & $0: 15: 30$ \\
MLFN 20 Nodes & 1.23 & $0: 16: 44$ \\
\hline
\end{tabular}

${ }^{a}$ The sample of the 28 trained samples and 7 tested samples were detected at the same time with the software auto-stopped

Therefore, it can be concluded that the ANN model derived from the GRNN model is a more suitable and reliable than the ANN model derived from the MLFN model in distinguishing old peels and new peels.

\section{GRNN model validation}

In order to determine the robustness of the GRNN model, $50 \%$ of the samples were set as the trained set and the rest were as the tested set. To ensure the persuasiveness of the test, experiments of the GRNN model were done 20 times, and each trained model was based on a different composition of samples. The average accuracy of both the old and new peels are relatively high (Table 3 ). In addition, according to the tested data of the GRNN model, the accuracy rates of the samples are mostly $100 \%$. This reveals that the judging processes of the characteristics of the old and new peels are robust and accurate. According to statistical analysis, all components had significant

Trop J Pharm Res, November 2015; 14(11): 2073 


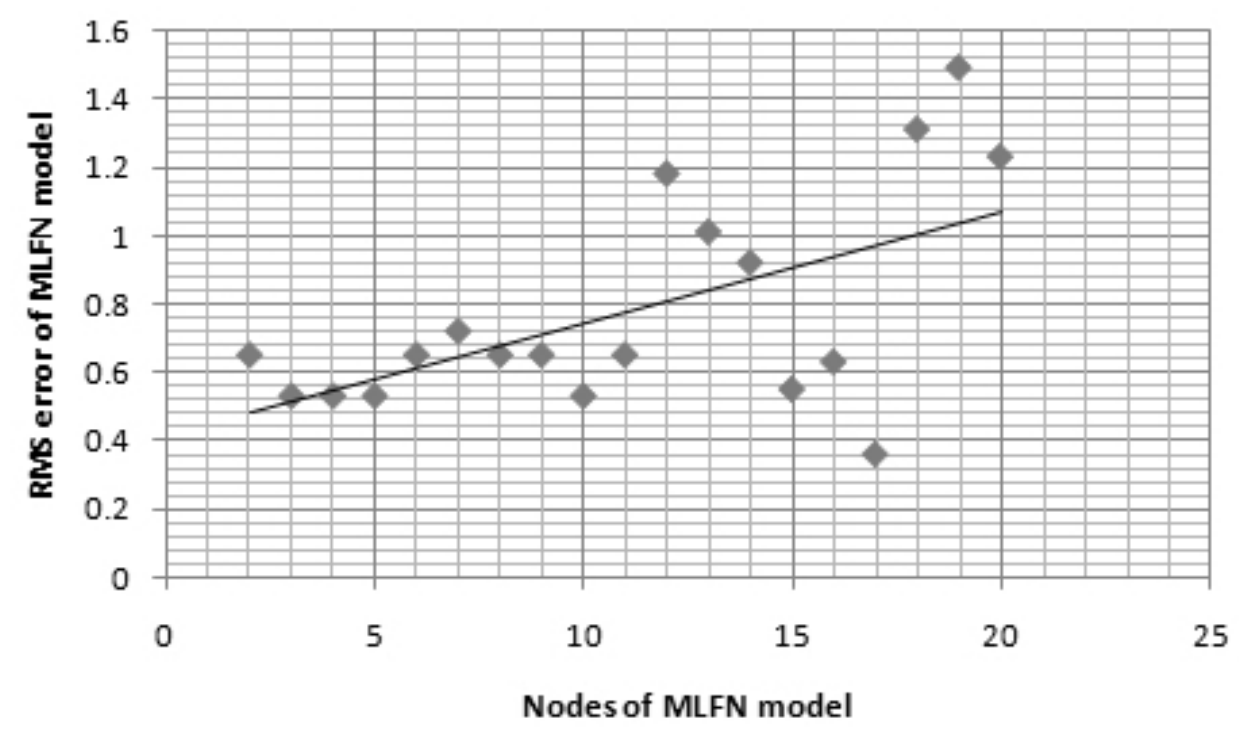

Fig 2: Comparison of the different MLFN models

contributions on these pattern recognition models. That is to say, every single independent variable for the sake of ensuring the robustness and precision of the model should not be ignored.

Table 3: Robustness of the MLFN-6 model

\begin{tabular}{lccc}
\hline Peel & $\begin{array}{c}\text { Sample } \\
\text { size }\end{array}$ & $\begin{array}{c}\text { Judging } \\
\text { digit }\end{array}$ & $\begin{array}{c}\text { Mean } \\
\text { correct } \\
\text { rate (\%) }\end{array}$ \\
\hline Old peel & 14 & 0 & $97 \%$ \\
New peel & 21 & 1 & $95 \%$ \\
\hline
\end{tabular}

\section{DISCUSSION}

Automated mass spectral deconvolution and identification system (AMDIS) was used to take chromatograms of complex samples to identify possible components [4] and these were widely applied in processing GC-MS data [5-9]. This identified automatically possible component peaks within the GC-MS data set in XPCR in this study. In previous studies, quality control and discrimination were done by developing analytical methods or coupled with some chemometric methods [10-12]. In the analysis by PCR (Polymerase Chain Reaction), studies on discrimination were about molecular authentication [13] and fingerprints [14]; most researches hammered at developing determining techniques [15-17]. The content of the components in the oil existed in a non-linear relationship and so ANNs can be used to analyze this relationship. The new analytical method may be used to distinguish between types of other Chinese herbs containing complex compounds.

\section{CONCLUSION}

A new and reliable method has been developed to distinguish between the characteristics of the old and new peels of XPCR accurately using ANN models. The results reveal that GRNN model, which is based on the comparison with MFLN, is robust and reliable. Neural networks can utilize actual data to differentiate old and new peels and can thus be applied to determine the specific storage times of old peels.

\section{ACKNOWLEDGEMENT}

The authors are grateful for the financial support extended by Natural Science Foundation of China (no. 81102804) and Education Department of the Sichuan Province, China (no. 10ZC051).

\section{REFERENCES}

1. Zhou X, Huang QH, Mo YY, Liao SM. Analysis on the volatile oil of Xinhui Pericarpium Citri Reticulatae in different years by GC/MS. Chin Med Mat 2009; 32: 24-26.

2. Wang J, Chen HP, Liu YP, Liu R, Fan DQ. Chemical variability of essential oils in Xinhui Citri Reticulatae Pericarpium stored in different years. Lishizhen Med Mater Med Res 2013; 24: 2831-2834.

3. Ebrahimzadeh A, Ranaee V. Control chart pattern recognition using an optimized neural network and efficient features. ISA transactions 2010; 49: 387393.

4. Asu JK, Bhattacharyya D, Kim T. Use of Artificial Neural Network in Pattern Recognition. International Journal 
of Software Engineering and Its Applications 2010; 4: 23-33.

5. Li W, Sun H, Wu B. Application of GC/MS/AMDIS in Forensic analyticalal Toxicology. Am J Foren Med Path 2007; 3: 11.

6. Carroll AJ, Badger MR, Millar AH. The Metabolome Express Project: enabling web-based processing, analysis and transparent dissemination of GC/MS metabolomics datasets. BMC Bioinformatics 2010; 11: 376.

7. Cunha SC, Faria MA, Fernandes JO. Determination of patulin in apple and quince products by GC-MS using 13C5-7 patulin as internal standard. Food Chem 2009; 115: 352-359.

8. Mihaleva VV, Verhoeven HA, De Vos RCH, Hall RD, Van Ham RCHJ. Automated procedure for candidate compound selection in GC-MS metabolomics based on prediction of Kovats retention index. Bioinformatics 2009; 25: 787-794.

9. Taylor J, King RD, Altmann T, Fiehn O. Application of metabolomics to plant genotype discrimination using statistics and machine learning. Bioinformatics 2002; 18: S241-S248.

10. Xiang Z, Wang X, Cai X, Zeng S. Metabolomics study on quality control and discrimination of three Curcuma species based on gas chromatograph-mass spectrometry. Phytochem Analysis 2011; 22: 411 418.

11. Bertrams J, Kunz N, Müller M, Kammerer D, Conrad Stintzing F. Phenolic compounds as marker compounds for botanical origin determination of
German propolis samples based on TLC and TLCMS.J Appl Bot Food Qual 2013; 86: 143-153.

12. Devendran G, Balasubramanian U. Qualitative phytochemical screening and GC-MS analysis of Ocimum sanctum L. leaves. Asian J of Plant Sci \& Res 2011; 1: 44-48.

13. Wang H, Kim MK, Kim YJ, Lee HN, Jin H, Chen J, Yang DC. Molecular authentication of the Oriental medicines Pericarpium Citri Reticulatae and Citri Unshius Pericarpium using SNP markers. Gene 2012; 494: 92-95.

14. Tistaert C, Thierry L, Szandrach A, Dejaegher B, Fan G, Frederich M, Vander Heyden Y. Quality control of Citri reticulatae pericarpium: Exploratory analysis and discrimination. Anal Chimacta 2012; 705: 111-122.

15. Xu G, Li X. Analysis of Volatile Constituents in Herbal Pair Pericarpium Citri Reticulatae-Fructus Aurantii and Its Single Herb by GC-MS and HELP Method. Res and Pr on Chin Med 2011; 3: 6.

16. Zheng GD, Zhou P, Yang H, Li YS, Li P, Liu EH. Rapid resolution liquid chromatographyelectrosprayionisation tandem mass spectrometry method for identification of chemical constituents in Citri Reticulatae Pericarpium. Food Chem. 2013; 136: 604-611.

17. Li T, Li X, Zhang M, Jiang C, Hu L, Yang X. Development and Validation of RP-HPLC Method for the Simultaneous Quantification of Seven Flavonoids in Pericarpium Citri reticulatae. Food Anal Method 2014; 7: 89. 